\title{
Desenvolvimento e calibração de guias de onda para TDR
}

\author{
Camilo de L. T. de Andrade ${ }^{1}$, Édio L. da Costa $^{2}$ \& Paulo E. P. de Albuquerque ${ }^{3}$
}

\author{
1 Embrapa Milho e Sorgo, CP 151, CEP 35701-970, Sete Lagoas, MG. Fone: (31) 3779-1000, Fax: (31) 3779-1088. \\ E-mail: camilo@cnpms.embrapa.br (Foto) \\ 2 EPAMIG. CP 12, CEP 39440-000, Janaúba, MG. Telefax: (38) 821-2160. E-mail: epamig@al.connect.com.br \\ ${ }^{3}$ Embrapa Milho e Sorgo
}

Protocolo $114-8 / 8 / 2002$ - Aprovado em 3/2/2003

\begin{abstract}
Resumo: A medição da umidade do solo é crucial para a pesquisa e o manejo de irrigação. Entre os métodos mais recentes de determinação da umidade está o da reflectometria no domínio do tempo (TDR) que se baseia na correlação entre a capacidade dielétrica do solo e a sua umidade. A obtenção do perfil de umidade do solo requer a utilização de um grande número de guias de onda, cujo custo ainda é elevado. Por outro lado, as curvas de calibração relacionando umidade com a capacidade dielétrica do solo foram desenvolvidas para outros meios porosos, solos desestruturados e solos de países com clima temperado. $\mathrm{O}$ objetivo deste trabalho foi construir uma guia de onda com materiais facilmente encontrados no mercado nacional e calibrá-la para solos tropicais. As curvas de calibração para o Neossolo quartzarênico e Latossolo Vermelho são distintas e diferentes dos modelos apresentados na literatura. A modificação de um modelo matemático, fisicamente embasado, permitiu a geração de curvas de calibração com ajuste adequado, porém independentes para cada tipo de solo.
\end{abstract}

Palavras-chave: instrumentação, umidade do solo, irrigação

\section{Development and calibration of TDR wave guides}

\begin{abstract}
Soil-water content measurement is critical for irrigation research and management. Time domain reflectometry (TDR) is among the newly developed methods for determination of soil-water content which relies on the relation between the soil dielectric property and water content. In order to obtain a soil water content profile, a large number of wave guides are necessary which raises the cost. On the other hand, calibration curves relating dielectric capacity to soil-water content have been developed mainly for soils of temperate regions. The objectives of this work were the development of a buriable wave guide using available materials and its calibration for tropical soils. Calibration curves developed for Psament and Haplustox soils are distinct and different from those found in the literature. A modification of a physically-based mathematical model fits well to the data of each soil type independently.
\end{abstract}

Key words: instrumentation, water content, irrigation

\section{INTRODUÇÃO}

A medição do potencial da água e da umidade do solo é essencial para a pesquisa e para o manejo racional da irrigação. Entre os métodos de medição da umidade do solo desenvolvidos recentemente está o da reflectometria no domínio do tempo (TDR), cujo princípio de funcionamento se baseia na relação existente entre a propriedade dielétrica (permissividade) e a umidade do solo. Uma descrição detalhada do princípio de funcionamento desse tipo de equipamento é apresentada na literatura (Soilmoisture, 1990; Or \& Wraith, 1997).

A relação entre a umidade e a capacidade dielétrica de um solo mineral é descrita por uma equação empírica desenvolvida por Topp et al. (1980):

$$
\theta=-5,310^{-2}+2,9210^{-2} \varepsilon_{\mathrm{b}}-5,510^{-4} \varepsilon_{\mathrm{b}}^{2}+4,310^{-6} \varepsilon_{\mathrm{b}}^{3}
$$

em que $\theta$ é a umidade do solo $\left(\mathrm{m}^{3} \mathrm{~m}^{-3}\right)$ e $\varepsilon_{\mathrm{b}}$ é a capacidade dielétrica (adimensional) do meio poroso, que engloba sólidos, água e ar. Os coeficientes da Eq. 1 são empíricos, ajustados para meios porosos artificiais e solos minerais de países temperados, numa faixa de umidade de 0 a $0,5 \mathrm{~m}^{3} \mathrm{~m}^{-3}$. Nos anos 90, outro modelo fisicamente embasado foi proposto por Roth et al. (1990):

$$
\theta=\frac{\varepsilon_{\mathrm{b}}^{\beta}-(1-\mathrm{n}) \varepsilon_{\mathrm{s}}^{\beta}-\mathrm{n} \varepsilon_{\mathrm{a}}^{\beta}}{\varepsilon_{\mathrm{w}}^{\beta}-\varepsilon_{\mathrm{a}}^{\beta}}
$$


em que $\varepsilon_{\mathrm{s}}, \varepsilon_{\mathrm{a}}, \varepsilon_{\mathrm{w}}$ são, respectivamente, as capacidades dielétricas da fase sólida, gasosa e líquida do solo, $n$ é a porosidade do solo $\left(\mathrm{m}^{3} \mathrm{~m}^{-3}\right)$ e $\beta$ é um parâmetro adimensional relacionado com a geometria do meio poroso. A capacidade dielétrica da fase sólida do solo, da água e do ar, é conhecida e alguns autores têm recomendado o valor de 0,5 para o parâmetro $\beta$.

O desempenho desse tipo de equipamento e a aplicabilidade das equações recomendadas pelo fabricante, e descritas na literatura vêm sendo avaliados em condições tropicais (Conciani et al., 1997; Pessoa \& Moraes, 1997; Silva, 1998; Pessoa et al., 2000; Souza et al., 2000; Coelho et al., 2001; Tommaselli \& Bachi, 2001). Os estudos demonstraram que o equipamento se presta para o monitoramento da umidade (Conciani et al., 1997; Pessoa \& Moraes, 1997; Silva, 1998; Souza et al., 2000; Tommaselli \& Bachi, 2001) ou do potencial da água no perfil do solo (Pessoa et al., 2000). Todavia, as equações recomendadas não são adequadas para alguns solos do Brasil, necessitando de calibrações locais, e mesmo individuais, para cada tipo de solo (Silva, 1998; Coelho et al., 2001; Tommaselli \& Bachi, 2001).

O efeito de propriedades como textura, densidade, teor de matéria orgânica, composição mineral e, também, da temperatura nas propriedades dielétricas do solo foi estudado por vários autores (Dasberg \& Hopmans, 1992; Persson \& Berndtsson, 1998; Jacobsen \& Schjonning, 1993; Halm et al.; 1999; Ponizovsky et al., 1999; Tomer et al., 1999; Coelho et al., 2001; Seyfried \& Murdock, 2001; Tommaselli \& Bachi, 2001).

Há, basicamente, três tipos de guia de onda que podem ser empregados para monitorar a umidade do solo: 1) de duas hastes grossas, cuja conexão com o cabo não é fixa, permitindo que se instalem hastes em vários pontos e se utilize a mesma conexão para leitura em vários locais (Soilmoisture, 1990);2) de três ou duas hastes finas, cuja conexão com o cabo é permanente, podendo ser enterrada na profundidade desejada do perfil do solo (Soilmoisture, 1990); 3) de haste segmentada, que é inserida verticalmente no perfil do solo (Conciani et al., 1997; Pessoa \& Moraes, 1997; Souza et al., 2000). O primeiro tipo é empregado para monitorar a umidade na superfície, enquanto os dois últimos se prestam para monitorar a umidade no perfil do solo. Devido ao custo elevado para o monitoramento em várias profundidades e em diversos pontos, guias de onda estão sendo confeccionadas localmente, empregando-se materiais de fácil obtenção (Cassel et al., 1994; Conciani et al., 1997; Souza et al., 2000; Coelho et al., 2001). Há, portanto, a necessidade de se produzir, a custo mais baixo, guias de onda para o monitoramento da umidade no perfil do solo.
Este trabalho teve como objetivo confeccionar, com materiais nacionais, e calibrar uma guia de onda para a TDR, modelo TRASE, fabricada pela Soil Moisture ${ }^{1}$.

\section{MATERIAL E MÉTODOS}

A calibração foi feita em campo, em um Latossolo Vermelho de Sete Lagoas, MG, e em um Neossolo quartzarênico do Projeto Jaíba, MG, cujas características são apresentadas na Tabela 1. Utilizou-se no estudo um equipamento TDR, modelo TRASE, pertencente à EPAMIG (Empresa de Pesquisa Agropecuária do Estado de Minas Gerais).

Guias de onda de três hastes paralelas, espaçadas entre si $1,5 \mathrm{~cm}$ e com $15 \mathrm{~cm}$ de comprimento, foram confeccionadas no Laboratório de Física do Solo da EMBRAPA Milho e Sorgo, empregando-se materiais nacionais. As hastes da guia foram construídas utilizando-se varetas de solda para aço inoxidável, tipo 308L, diâmetro de $3,25 \mathrm{~mm}$. Um cabo coaxial do tipo RG-58, $50 \mathrm{ohms}$, flexível, foi utilizado para conectar a guia de onda ao equipamento. Em uma de suas extremidades, foi fixado, por pressão, um conector tipo BNC. A parte externa do cabo coaxial foi dividida para conectar-se nas duas hastes externas e a parte interna foi soldada à haste central da guia. Um capacitor de $3,9 \mathrm{pF}$, soldado entre duas das três hastes, foi necessário para marcar, eletronicamente, o início da guia de onda. $\mathrm{O}$ algoritmo interno da TDR necessita de um pico no início e de uma elevação no final da curva de voltagem versus tempo, para separar a guia de onda propriamente dita do restante do cabo (Soilmoisture, 1990). A função do capacitor foi gerar o pico no início da onda, sem o qual o algoritmo não consegue calcular corretamente a capacidade dielétrica do meio. Para manter as hastes separadas de uma distância fixa sobre a conexão hastescabo, fundiu-se um bloco de resina de poliéster tipo T-208, com respectivo catalisador (Figura 1).

A calibração foi feita em campo, instalando-se guias de onda na posição horizontal, nas profundidades de 20 e $40 \mathrm{~cm}$ valendo-se, para isto, de pequenas trincheiras abertas minutos antes. Na seqüência, leituras foram feitas com a TDR e, logo em seguida, amostras de solo não-deformado foram retiradas em cada profundidade, para determinação da umidade pelo método gravimétrico. Leituras e amostragens foram realizadas em diversas condições de umidade do solo.

${ }^{1}$ Referência à marca registrada não constitui endosso por parte dos autores e instituições envolvidas no trabalho

Tabela 1. Características físico-hídricas e concentração de matéria orgânica para os solos estudados

\begin{tabular}{|c|c|c|c|c|c|c|c|c|c|c|}
\hline \multirow{3}{*}{$\begin{array}{l}\text { Prof. } \\
\mathrm{cm}\end{array}$} & \multicolumn{2}{|c|}{ Densidade } & \multirow{2}{*}{$\begin{array}{c}\text { Capacidade } \\
\text { de Campo }\end{array}$} & \multirow{2}{*}{$\begin{array}{c}\text { Ponto de } \\
\text { Murcha } \\
\text { Permanente }\end{array}$} & \multirow{2}{*}{$\begin{array}{c}\text { Porosidade } \\
\text { Total }\end{array}$} & \multicolumn{5}{|c|}{ Composição Granulométrica } \\
\hline & Partículas & Solo & & & & $\begin{array}{c}\text { Areia } \\
\text { Grossa }\end{array}$ & $\begin{array}{c}\text { Areia } \\
\text { Fina }\end{array}$ & Silte & Argila & $\begin{array}{l}\text { Matéria } \\
\text { Orgânica }\end{array}$ \\
\hline & \multicolumn{2}{|c|}{$\mathrm{Mg} \mathrm{m}^{-3}$} & \multicolumn{3}{|c|}{$\mathrm{m}^{3} \mathrm{~m}^{-3}$} & \multicolumn{4}{|c|}{$\mathrm{g} \mathrm{kg}^{-1}$} & $\mathrm{~g} \mathrm{~g}^{-1}$ \\
\hline \multicolumn{11}{|c|}{ Latossolo Vermelho } \\
\hline $0-10$ & 2,56 & 0,87 & 0,346 & 0,234 & 0,658 & 50 & 30 & 390 & 530 & 42 \\
\hline $10-26$ & 2,56 & 1,08 & 0,413 & 0,298 & 0,579 & 50 & 30 & 240 & 680 & 34 \\
\hline $26-49$ & 2,53 & 0,95 & 0,385 & 0,278 & 0,623 & 50 & 40 & 180 & 730 & 31 \\
\hline \multicolumn{11}{|c|}{ Neossolo quartzarênico } \\
\hline $0-10$ & 2,61 & 1,46 & 0,089 & 0,050 & 0,438 & 583 & 306 & 12 & 99 & 6 \\
\hline $10-30$ & 2,63 & 1,54 & 0,090 & 0,051 & 0,416 & 579 & 324 & 7 & 90 & 4 \\
\hline $30-50$ & 2,60 & 1,48 & 0,113 & 0,064 & 0,429 & 499 & 379 & 12 & 110 & 2 \\
\hline
\end{tabular}




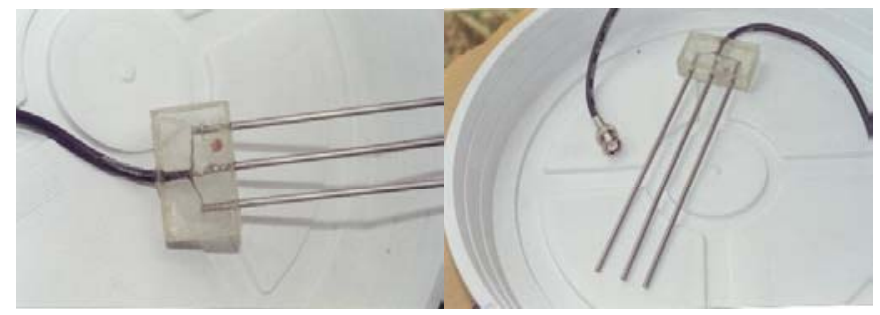

Figura 1. Detalhe do capacitor soldado entre duas hastes e do conector da guia de onda

Os modelos de Topp et al. (1980) e de Roth et al. (1990) foram testados para descrever a relação entre umidade e capacidade dielétrica do solo. O modelo de Roth et al. (1990) foi também modificado, incluindo nele uma constante, $\alpha$, e permitindo o ajuste do parâmetro $\beta$ :

$$
\theta=\alpha+\frac{\varepsilon_{\mathrm{b}}^{\beta}-(1-\mathrm{n}) \varepsilon_{\mathrm{s}}^{\beta}-\mathrm{n} \varepsilon_{\mathrm{a}}^{\beta}}{\varepsilon_{\mathrm{w}}^{\beta}-\varepsilon_{\mathrm{a}}^{\beta}}
$$

Ferramentas de ajuste de modelos não-lineares de planilhas eletrônicas foram empregadas na determinação dos parâmetros $\alpha$ e $\beta$.

\section{RESULTADOS E DISCUSSÃO}

Dados de umidade versus capacidade dielétrica, para os dois tipos de solo, bem como as curvas de calibração para as guias-de-onda, são apresentados na Figura 2. Nota-se que os valores de umidade e de capacidade dielétrica são muito distintos para os dois solos, contrariando o conceito de calibração universal preconizado pelo fabricante da TDR (Soilmoisture, 1990) e também discutido na literatura (Roth et al., 1992; Coelho et al., 2001; Tommaselli \& Bachi, 2001). Valores de capacidade dielétrica entre 2,5 e 7,5 correspondem a valores muito menores de umidade no Neossolo, que no Latossolo. O efeito da textura na estimativa da umidade com TDR foi também observado por outros pesquisadores (Dasberg \& Hopmans, 1992; Jacobsen \& Schjonning, 1993; Tommaselli \& Bachi, 2001; Ponizovsky et al., 1999; Tomer et al., 1999; Seyfried \& Murdock, 2001).

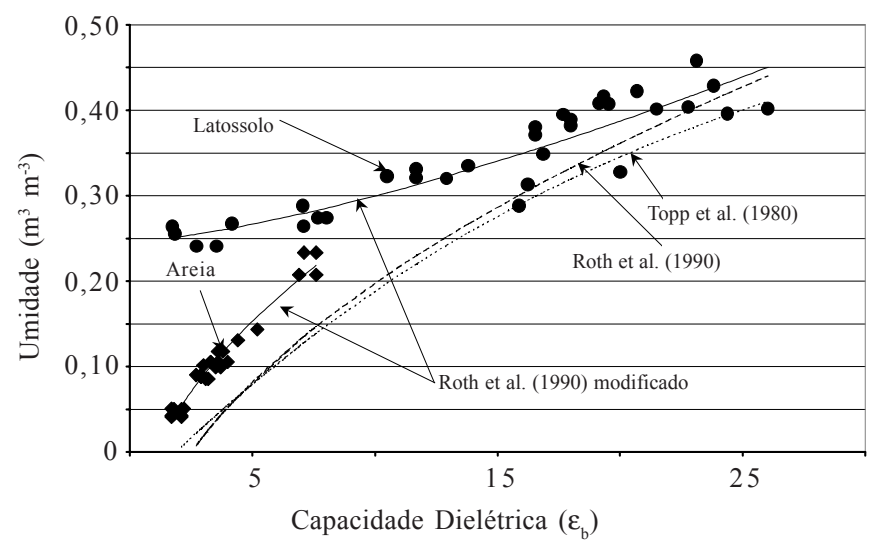

Figura 2. Umidade do solo em função da capacidade dielétrica para dois tipos de solo e curvas de calibração para guias-deonda confeccionadas em laboratório
Os modelos de Topp et al. (1980) e de Roth et al. (1990) subestimaram a umidade nos dois tipos de solo, conforme também observaram outros autores (Gervasio \& Silva, 1999; Tomer et al., 1999; Tomaselli \& Bachi, 2001). Em estudos realizados em materiais de solo com textura diferente, verificouse que a relação entre a capacidade dielétrica e a umidade do solo é melhor descrita com modelos exponenciais (Coelho et al., 2001) ou polinomiais (Tommaselli \& Bachi, 2001). No Latossolo, as diferenças são maiores na faixa de baixa umidade, na qual a capacidade dielétrica da fase líquida não é predominante. Pode estar havendo interferência dos óxidos de ferro e de alumínio na capacidade dielétrica dos solos tropicais, o que requer estudos mais aprofundados. Em trabalho realizado por Halm et al. (1999), a adição de 6\% em peso de magnetita na areia lavada aumentou consideravelmente as leituras da umidade do solo, obtidas com TDR.

O modelo de Roth et al. (1990) modificado (Eq. 3) foi adequado para ambos os solos (Figura 2 e Tabela 2), porém com parâmetros de ajuste diferentes para cada um dos solos estudados.

Tabela 2. Parâmetros do modelo de Roth original (Roth et al., 1990) e modificado, ajustados para os dados de umidade versus capacidade dielétrica para os solos estudados

\begin{tabular}{|c|c|c|c|}
\hline \multirow[b]{2}{*}{ Parâmetros } & \multicolumn{2}{|c|}{ Roth-Modificado } & \multirow{2}{*}{$\begin{array}{l}\text { Roth- } \\
\text { Original }\end{array}$} \\
\hline & $\begin{array}{c}\text { Neossolo } \\
\text { Quartzarênico }\end{array}$ & $\begin{array}{l}\text { Latossolo } \\
\text { Vermelho }\end{array}$ & \\
\hline$\beta$ & 0,4960 & 1,4020 & 0,5000 \\
\hline$\alpha$ & 0,0707 & 0,2533 & 0,0000 \\
\hline $\mathrm{n}$ & 0,4190 & 0,6170 & 0,4190 \\
\hline Soma Quadrado Desvios & 0,0033 & 0,0221 & \\
\hline Variância & 0,0028 & 0,0033 & \\
\hline Número de Pontos & 29 & 33 & \\
\hline $\mathrm{R}^{2}$ & 0,9590 & 0,7981 & \\
\hline
\end{tabular}

O valor de $\beta$ para o Neossolo é praticamente igual ao proposto por Roth et al. (1990) para um meio poroso isotrópico; entretanto, o valor de $0,07 \mathrm{~m}^{3} \mathrm{~m}^{-3}$, da constante $\alpha$ ajustada, desloca a curva para cima. No caso do Latossolo, o valor de $\beta$ é ainda maior, estando fora dos limites de -1 e 1, definidos por Roth et al. (1990).

Os valores da constante $\alpha$ para ambos os solos estão próximos de valores médios da umidade do solo no ponto de murcha permanente $(-1500 \mathrm{kPa})$. Uma abordagem semelhante com que se tentou associar o termo independente de um modelo polinomial, com outras propriedades do solo já foi explorada por Tommaselli \& Bachi (2001). Este fato é importante no aprofundamento dos estudos visando a uma equação generalizada para solos minerais. A inclusão, no modelo, de outros parâmetros relacionados à textura, porosidade, densidade e matéria orgânica, já foi experimentada em outros trabalhos (Jacobsen \& Schjonning, 1993; Ponizovsky et al., 1999; Tommaselli \& Bachi, 2001).

\section{CONCLUSÕES}

1. A confecção de guias de onda para TDR modelo TRASE, utilizando-se materiais nacionais, foi possível, o que possibilita 
ampliar a utilização desse equipamento em pesquisa e manejo de irrigação.

2. O comportamento da relação entre umidade e capacidade dielétrica do solo foi diferente para Neossolo quartzarênico e Latossolo Vermelho e requereu curvas de calibração distintas.

3. Os modelos existentes na literatura não descrevem corretamente essa relação para os solos estudados.

4. A modificação do modelo de Roth original permitiu o ajuste de curvas de calibração separadas para cada um dos solos.

\section{LITERATURA CITADA}

Cassel, D.K.; Kachanoski, R.G.; Topp, G.C. Practical considerations for using TDR cable tester. Soil Technology, CremlingenDestedt, v.7, n.2, p.113-126, 1994.

Coelho, E.F.; Andrade, C.L.T.; Or, D.; Lopes, L.C.; Souza, C.F. Desempenho de diferentes guias de ondas para uso com o analisador de umidade TRASE. Revista Brasileira de Engenharia Agrícola e Ambiental, Campina Grande v.5, n.1, p.81-87, 2001.

Conciani. W.; Carneiro, B.J.; Soares, M.M; Herrmann, P.S.P.; Crestana, S. Emprego de TDR com sondas multiastes segmentadas para medida de umidade de um perfil de solo. In: Simpósio Nacional de Instrumentação Agropecuária, 1, 1996, São Carlos, Anais... Brasília: EMBRAPA-SPI, 1997. p.169-179.

Dasberg, S.; Hopmans, W. Time domain reflectometry calibration for uniformly and non uniformly wetted sandy and clayey loam soils. Soil Science Society of America Journal, Madison, v.56, p.1341-1345, 1992.

Gervasio, E.S.; Silva, E.L. Uso do instrumento TDR para determinação do teor de água em diferentes camadas de um Latossolo Roxo distrófico. Revista Brasileira de Engenharia Agrícola e Ambiental, Campina Grande, v.3, n.3, p.417-420, 1999.

Halm, D.; Gaiser, T.; Stahr, K. Does magnetite have an influence on measurements of water contents by time domain reflectometry (TDR). Journal of Plant Nutrition and Soil Science, Weinheim, v.162, n.6, p.661-665, 1999.

Jacobsen, O.H.; Schjonning, P. A. A laboratory calibration of time domain reflectometry for soil water measurement including effects of bulk density and texture. Journal of Hydrology, Amsterdam, v.151, p.147-157, 1993.

Or, D.; Wraith, J.M. Agricultural and environmental soil physics. Logan: Utah State University, 1997. 218p.

Persson, M.; Berndtsson, R. Texture and electrical conductivity effects on temperature dependency in time domain reflectometry. Soil Science Society of America Journal, Madison, v.62, n.4, p.887-893, 1998.
Pessoa, J.D.C.; Kanno, S.S; Carneiro, B.J.I.; Moraes, J.A.P.V. Avaliação da técnica de TDR para medida do potencial de sucção em um solo sob vegetação de cerrado. In: Simpósio Nacional de Instrumentação Agropecuária, 2, 1998, São Carlos, Anais... São Carlos: EMBRAPA, 2000. p.341-345.

Pessoa, J.D.C.; Moraes, J.A.P.V. Acompanhamento da umidade de um solo de cerradão com a técnica de TDR durante a transição entre as estações seca e chuvosa. In: Congresso Brasileiro de Ciência do Solo, 26, 1997, Rio de Janeiro. Anais... Rio de Janeiro: Sociedade Brasileira de Ciência do Solo, 1997. p.28-31. CD-Rom

Ponizovsky, A.A.; Chudinova, S.M.; Pachepsky, Y.A. Performance of TDR calibration models as affected by soil texture. Journal of Hydrology, Amsterdam, v.218, p.35-42, 1999.

Roth, C.H.; Malicki, M.A.; Plagge, R. Empirical evaluation of the relationship between soil dieletric constant and volumetric water content as the basis for calibration soil moisture measurements by TDR. Journal of Soil Science, Reading, v.43, n.1, p.1-13, 1992.

Roth, K.; Shulin, R.; Fluhler, H; Attinger, W. Calibration of time domain reflectometry for water content measurement using composite dielectric approach. Water Resources Research, Washington, v.26, n.10, p.2267-2273, 1990.

Seyfried, M.S.; Murdock, M.D. Response of a new soil water sensor to variable soil, water content and temperature. Soil Science Society of America Journal, Madison, v.65, n.1, p.28-34, 2001.

Silva, E.L. Determinação automática do teor de água em Latossolo Roxo distrófico com uso de reflectometria de onda. In: Congresso Brasileiro de Engenharia Agrícola, 27, 1998, Poços de Caldas, Anais... Lavras:UFLA/SBEA, 1998. p.154-156.

Soilmoisture Equipment Corp. TRASE operating instructions. Goleta, CA: Soilmoisture Equipment Corp., 1990. 110p.

Souza, C.F.; Matsura, E.E.; Testezlaf, R. Utilização de um equipamento TDR (Trase System I) com sonda multihaste segmentada para medir a umidade do solo. In: Simpósio Nacional de Instrumentação Agropecuária, 2, 1998, São Carlos, Anais... São Carlos: EMBRAPA, 2000. p.161-169.

Tomer, M.D.; Clothier, B.E.; Vogeler, I.; Green, S.A dielectricwater content relationship for sandy volcanic soils in New Zealand. Soil Science Society of America Journal, Madison, v.63, n.4, p.777-781, 1999.

Tommaselli, J.T.G.; Bachi, O.O.S. Calibração de um equipamento de TDR para medida de umidade de solos. Pesquisa Agropecuária Brasileira, Brasília, v.36, n.9, p.1145-1154, 2001.

Topp, G.C.; Davis, J.L.; Annan, A.P. Electromagnetic determination of soil water content: Measurements in coaxial transmission lines. Water Resources Research, Washington, v.16, p.574-582, 1980. 